\title{
EPIs para mulheres trabalhadoras da Construção Civil: oportunidade de debate no Design
}

\author{
Mayanne Camara Serra ${ }^{1}$;
}

Ivana Marcia Oliveira Maia²;

resumo:

Historicamente os postos de trabalho foram desenvolvidos por e para homens, principalmente os de ambientes operacionais como na Construção Civil, sendo tardia a inserção da mulher no mercado de trabalho. Desta forma, houve repercussões no desenvolvimento de Equipamentos de Proteção Individual (EPIs), que tendem a ser baseados majoritariamente nas características masculinas. Por esta razão, ainda persistem na atualidade diversos obstáculos quanto ao acesso e uso de EPIs adequados por mulheres trabalhadoras da Construção Civil, sendo isso uma questão não apenas nacional. A partir desta contextualização, sinteriza-se que este artigo é baseado em uma Revisão Sistemática de Literatura que tem como questão direcionadora o que se pode debater dos principais entraves relacionados aos EPIs enquanto produtos de proteção usados por mulheres na construção civil? Ademais, o objetivo principal é levantar inconsistências e desafios no que concerne aos EPIs voltados para o uso por trabalhadoras da Construção Civil. Portanto, a partir de revisão em três bases de dados, cinco publicações levantadas dos últimos dez anos deixam em destaque a inadequação antropométrica dos EPIs para mulheres, além da necessidade de ajustes nos produtos. Todavia, há outros desafios para as trabalhadoras da Construção Civil que podem fomentar debates oportunos no campo do Design.

palavras-chave:

Design. Equipamento de Proteção Individual. Trabalhadoras da Construção Civil.

Espaço reservado para organização do congresso.

\footnotetext{
${ }^{1}$ http://lattes.cnpq.br/8862313255315071

${ }^{2}$ http://lattes.cnpq.br/1520994716359117
} 


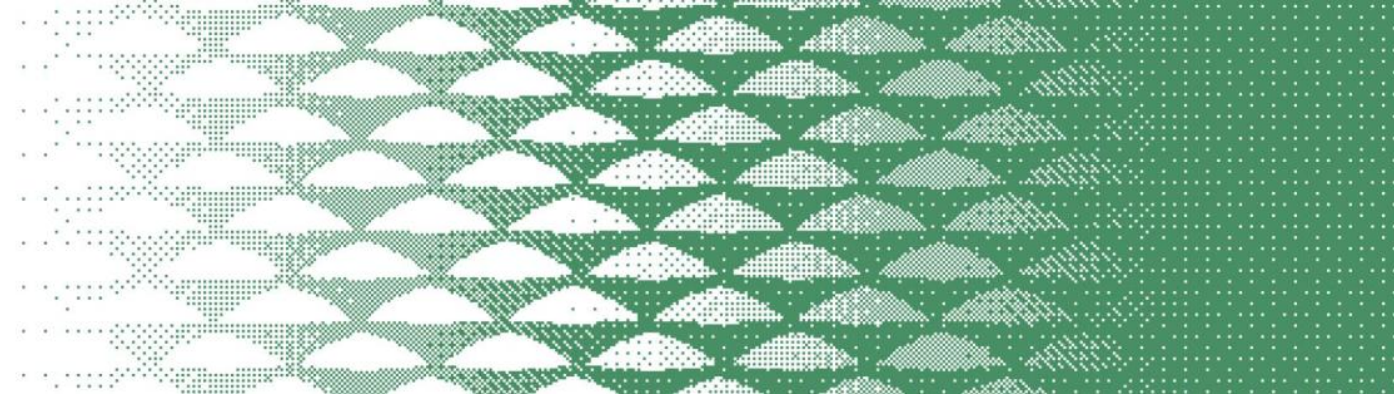

\section{Introdução}

Na prática do trabalho de forma geral, há diversos tipos de riscos de acidentes e adoecimentos que devem ser rigorosamente evitados. Para isso, os riscos devem ser eliminados através de medidas organizacionais e de engenharia, considerando os preceitos do campo da Saúde e Segurança do Trabalho (SST). Assim, devem ser implementados os chamados Equipamentos de Proteção Coletiva (EPCs) e, quando ainda não possível for evitar algum risco, devem ser fornecidos os Equipamentos de Proteção Individual (EPIs) aos trabalhadores.

Nacionalmente, os EPIs possuem como principal dispositivo de respaldo a Norma Regulamentadora de número 6 - NR 6. Conforme o conteúdo deste documento regulador, em Escola Nacional de Inspeção do Trabalho (ENIT, 2018), EPIs consistem em produtos ou dispositivos que são utilizados por trabalhadores de forma individualizada a fim de se resguardar de riscos à sua saúde e segurança. Ainda conforme a NR, há vários tipos de EPIs, dentre os quais há os voltados para proteção visual, facial, auditiva, respiratória, da cabeça, de mãos, de braços, de pernas, de pés e contra quedas.

Considerando o disposto, vale sublinhar que os EPIs são produtos tangíveis que são calçados, vestidos ou acoplados ao corpo do trabalhador em situações de risco de menor ou maior grau, que é o que se entende da leitura da NR 6. Em face disso, são inegáveis as hipóteses de desconfortos ou dificuldades no uso destes objetos. Desta maneira, tem-se uma demanda para o campo do Design, haja vista a essencialidade destes produtos que devem ser projetados e produzidos com direção à eficiência no seu uso. Em soma, há as interfaces com a Ergonomia por envolver aspectos antropométricos, ampliando o contexto interdisciplinar em volta dos EPIs.

Estima-se que oportunidades de estudos tendo os EPIs como objeto de pesquisa em Design estão na identificação de pontos de melhoria, que, por sua vez, podem ser materializados e disponibilizados para a sociedade. À vista disso, como se inspira do abordado por Wanderley et al. (2017), deve-se deixar em luz que o Design é social e há um papel de engajamento que deve ser repercutido nos âmbitos diversos para os cidadãos, tais como os da saúde, diretos humanos, na segurança do trabalho como aqui versado, dentre outros.

Com o tema dos EPIs, são numerosos os estudos que tocam na resistência à adesão ao uso no ambiente de trabalho, preponderando-se as queixas de dificuldades em torno desses produtos de proteção. Logo, infere-se que é bastante crítica a realidade do uso não conforme de EPIs. Todavia, se de forma difusa há entraves quanto ao uso e função de EPIs por trabalhadores, os desafios devem ser ainda maiores quando se coloca a usuária mulher no enfoque de análise. Isto porque a grande maioria dos postos de trabalho surgiu e se desenvolveram levando em conta apenas o homem como usuário no decorrer da história da sociedade.

Portanto, com os ambientes de trabalho se desenvolvendo com base em características masculinas, além da tardia inserção das mulheres no mercado de trabalho de forma global, não seria diferente que os EPIs fossem criados e melhorados sempre priorizando um usuário do gênero masculino. Por isso, ainda persistem implicações no uso de EPIs por mulheres mesmo com a modernidade, como se percebe de diversas pesquisas.

Del Castillo (2015), por exemplo, elenca vários EPIs demandantes de correções para o conforto no uso por mulheres devido ao fato de a maioria destes produtos ter sido projetada por e para homens. A autora destaca em seu estudo a ausência de roupas e produtos de proteção ocupacional projetadas especificamente para mulheres, em que luvas, calçados e calças prevalecem em termos de criticidade. Indo além, Del Castillo (2015) alerta sobre a dificuldade de EPIs para trabalhadoras grávidas.

Para complementar, Rosa e Quirino (2017) realizaram estudo voltado para a percepção sobre os postos de trabalho de mulheres que trabalhavam como operacionais na indústria e no setor da 


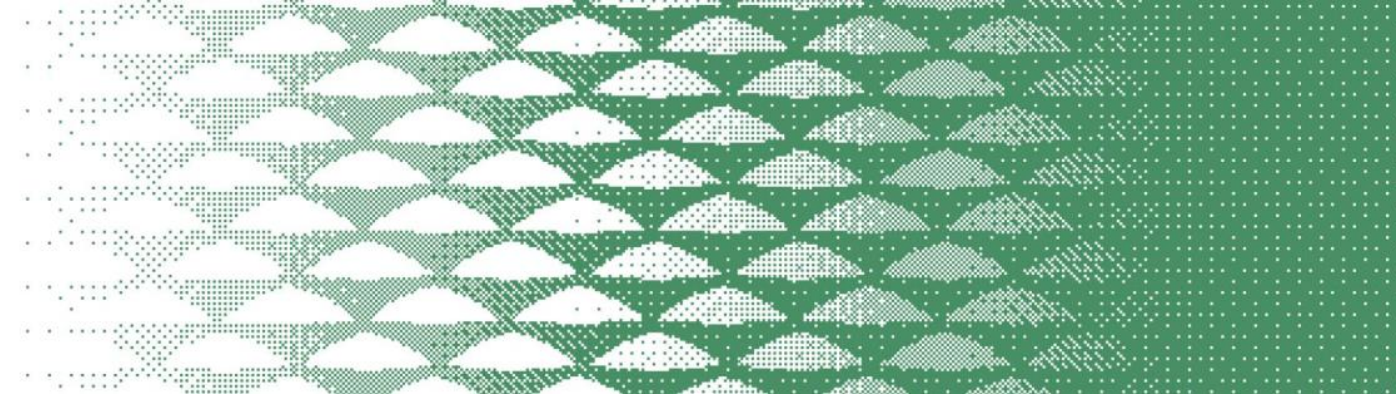

Construção. Desta forma, as autoras identificaram como entraves no uso de EPIs a adesão imposta ao uso de vestimentas ocupacionais masculinas e a baixa disponibilidade destas vestimentas para trabalhadoras gestantes. Dentre os EPIs masculinos que eram utilizados pelas trabalhadoras, estavam os jalecos, botinas e calças. Estes produtos foram referenciados como desconfortáveis e de largas dimensões pelas operárias. Neste aspecto, as autoras ressaltam o fornecimento de apenas luvas de tamanho médio ("M"), em que se entende que o intuito era obter um produto considerado "unissex". Porém, apesar do tamanho supostamente mediano do EPI, era reduzida a conformidade com as mãos das trabalhadoras.

Meireles, Veiga e Duarte (2016) apontam em seu referencial teórico que há na realidade registros oficiais de várias falhas no atendimento de EPIs quando se tem uma usuária em alguns campos de trabalho. Neste ponto, os autores realçam a não conformidade antropométrica em EPIs por carência de atualizações. Como exemplo, os autores explicitam sobre luvas e botas utilizadas como EPIs na realidade da Construção dos Estados Unidos, que se demostram como inadequadas para mulheres sob a ótica da Antropometria.

Aproveitando-se a menção sobre o termo "Antropometria", vale situar sua posição de destaque no âmago da Ergonomia. Conforme explicações de Panero e Zelnik (2008), a Antropometria consiste na ciência que versa sobre a especificação de medidas do corpo humano no intuito de gerar distinções individuais e grupais. Os autores ressaltam que esta ciência é incipiente e não exata, mas seus dados são ferramentas fundamentais para projetos, e alertam que o bom senso e os aspectos da criatividade não devem ser relegados a dados de tabelas.

Em complemento, no que tange à aplicação da Ergonomia e que faz referência à Antropometria, Dul e Weerdmeester (2012) abordam sobre a recomendação de que equipamentos, atividades e sistemas sejam projetados visando a coletividade. No entanto, como existem diferenças entre indivíduos em uma população, os autores deixam a orientação para que os projetos atendam a $95 \%$ dessa população. No caso, os demais $5 \%$ tendem a se relacionar a gestantes, obesos, pessoas de estatura muito baixa, idosos e pessoas com deficiência física, e, por isso, projetos específicos devem ser realizados visando esse percentual populacional.

Quando se discute sobre aspectos antropométricos sob a ótica de gênero, as diferenças individuais se ampliam sobremaneira. Para este assunto, recorre-se a Iida e Guimarães (2016), que leciona que a maior parte das medidas antropométricas de homens supera as medidas femininas; mas geralmente sendo excetuada a medida do quadril, que tende a ser maior nas mulheres. Quanto ao exposto, Messing e Chatigny (2018) mencionam que variações mais significativas nas distinções antropométricas masculinas e femininas dependem do segmento do corpo, pois, como exemplificado pelas autoras, a relevância de análise é mais considerável para a largura de mãos quando comparado com o tamanho da bacia.

Considerando as apreciações do parágrafo anterior, salienta-se que é impensável a obtenção de EPIs efetivamente femininos apenas com a redução de todas as medidas masculinas para fundamentar o projeto desses produtos. Portanto, é imperativo trazer para destaque as condições de segurança laboral investidas às mulheres por meio do uso de EPIs, principalmente em locais de trabalho historicamente masculinizados, como o da Construção Civil.

Com base no versado até aqui, tem-se a contextualização para a questão central que norteia este estudo e que traz os Equipamentos de Proteção Individual para a abordagem do Design. Essa questão representa a pergunta de pesquisa, que é: o que se pode debater dos principais entraves relacionados aos EPIs enquanto produtos de proteção usados por mulheres na construção civil? Com essa problemática sintetizada, apresenta-se que este estudo tem como objetivo principal levantar inconsistências e desafios no que concerne aos EPIs voltados para o uso por trabalhadoras da Construção Civil. 


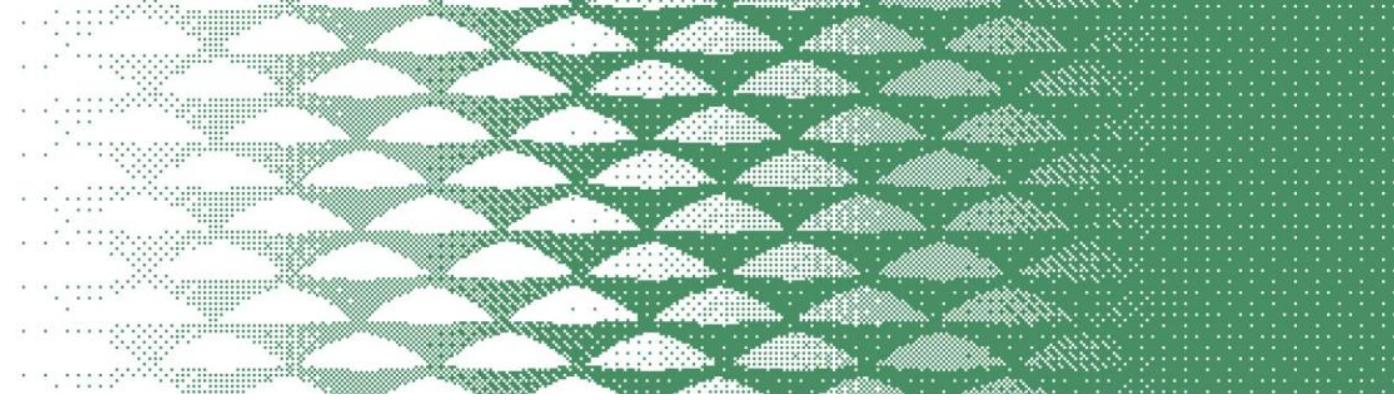

\section{Resultados e síntese}

Na pesquisa de Rocha (2015), trabalhadoras da construção participaram juntamente com mulheres de outros segmentos, como da metalurgia e confecção industrial. Embora as operárias construtoras não possuam enfoque exclusivo no estudo em descrição, a autora traz resultados pertinentes, em particular sobre saúde auditiva. Segundo a autora, o ruído, quedas e condições ergonomicamente inadequadas foram os riscos ocupacionais mais prevalecentes.

Ainda quanto ao estudo de Rocha (2015), é ressaltado que todas as trabalhadoras da construção referiram o uso de EPI. Entretanto, o setor em questão foi o considerado como mais ruidoso, seguido do setor da metalurgia. Com esse quadro, infere-se que, embora ocorra o uso de EPIs de proteção auditiva, não há eficiência no resguardo da audição. Neste aspecto, a autora deixa alerta para as consequências do excesso de ruído, pois indica como exemplo estudos com achados relacionados a problemas em fetos por excessos de exposição de trabalhadoras gestantes ao ruído.

No estudo americano de Wagner, Kim e Gordon (2013), que envolve a participação de carpinteiras, mulheres eletricistas, dentre outras profissionais do setor da construção, há a análise da relação entre os EPIs-roupas de trabalho com a autoeficácia e satisfação do trabalho por mulheres que atuam no ramo da temática. Desta forma, é demonstrado que essa relação é positiva; isto é, que a autoeficácia e a satisfação do trabalho podem ser ampliadas através do uso de EPIs e vestimentas ocupacionais adequadamente projetados e acessíveis. Indo além desta resolução, os autores evidenciam vários aspectos oportunos que, segundo eles, podem gerar desdobramentos por meio de critérios projetuais específicos visando EPI para mulheres na Construção Civil. Além disso, apoiando-se em outras pesquisas, os autores explicam que existem disponíveis muitos estudos que enfocam os EPIs, porém poucos deles abordam o aspecto das características corporais das necessidades específicas por gêneros, principalmente o feminino.

De acordo com Wagner, Kim e Gordon (2013), o mercado de EPI específicos para mulheres melhorou nas últimas décadas, porém ainda persistem limitações e há espaços de melhoria para esses produtos, valendo isso não apenas para o setor da construção. Dentre os EPIs, os autores apontam os cintos de segurança, que requerem o ajuste de tamanho devido às altas discrepância antropométrica entre mulheres e homens, além de luvas e roupas médias que são fabricadas para homens, mas também fornecidos às trabalhadoras. Os autores ressaltam que todos esses pontos afetam a produtividade das mulheres. Para agravar, conforme as linhas discorridas pelos autores, entende-se que não somente os EPIs devem ser adequados para mulheres, pois também há a referência às ferramentas manuais, as quais tendem a apresentar cabos muito grossos para as mãos femininas, por exemplo.

Onyebeke et al. (2016) trazem como reiteração que a maior parcela dos EPIs utilizados na Construção Civil foram produzidos em função do usuário do gênero masculino, ficando a Antropometria de mulheres não contemplada. Neste estudo, os autores entrevistaram trabalhadoras de carpintaria com o uso da técnica de grupo focal, em que as integrantes apresentavam uma média de tempo de atuação na Construção Civil em torno de 15 anos. Portanto, percebe-se que a amostra dos autores era formada por mulheres com considerável tempo de trabalho como operárias e, com isso, a maioria poderia colaborar com diversas experiências relacionadas a EPIs em contexto laboral.

Através da pesquisa que desenvolveram, Onyebeke et al. (2016) receberam diversas contribuições fornecidas pelas trabalhadoras sobre os EPIs apresentarem deficiência em ajustes. Desta maneira, foram relatados que botas, luvas, roupas ocupacionais e coletes de segurança sempre demandavam que as usuárias realizassem algum tipo de ajuste durante o uso. Como principal razão para isso, os EPIs mencionados pelas participantes eram caracterizados como de grandes dimensões. Diante do descrito e retomando o aspecto do tempo de trabalho na média de 15 anos pelas trabalhadoras 


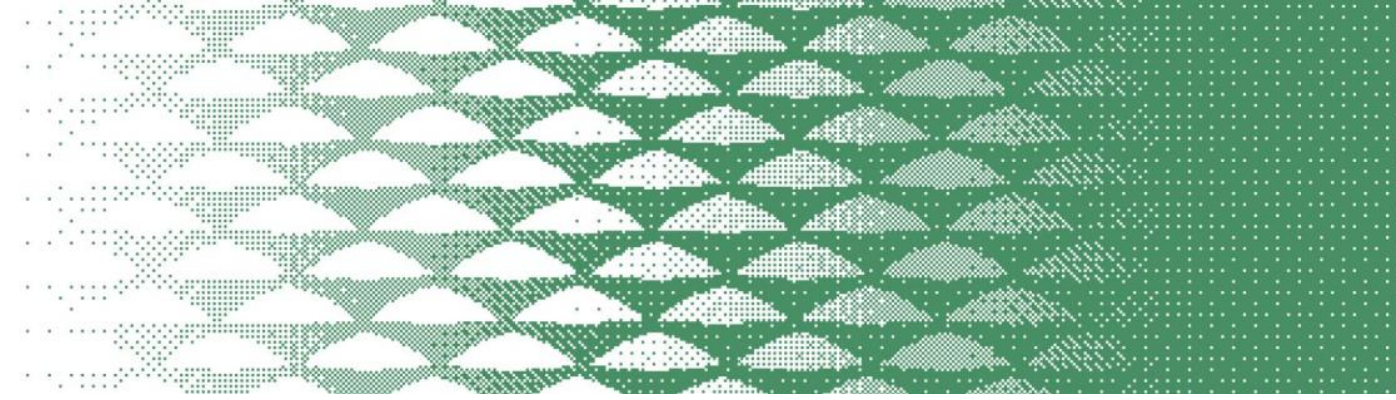

participantes do estudo, chama-se a atenção para o expressivo período em que estas permaneceram sob riscos devido ao uso de EPIs inadequados.

O enfoque da pesquisa de Min (2015) estava na imagem de trabalhadoras retratadas em cenários e comercialização de EPIs. A autora delimitou seu estudo às chamadas trabalhadoras de "colarinho azul" ("blue-collar"), que se referem àquelas que atuam no campo operacional da construção, metalurgia e afins. Neste estudo, a autora constatou que as imagens veiculadas para comercializar EPIs representam mulheres em desvantagem em relação aos homens, como se tivessem baixa capacidade para realizar as suas funções ao propagar fotografias de mulheres em trabalhos mais leves.

Outros resultados de Min (2015) demonstram a disponibilização de EPIs no mercado nas cores rosas e similares, reduzindo a especificação dos produtos de proteção para mulheres ao aspecto estético, sendo que as próprias trabalhadoras repudiavam isso. Além disso, poucas opções de tamanhos e ajustes para o público feminino eram apresentadas por empresas fornecedoras, quando comparado às opções mais amplas de EPIs para usuários homens. Dentre esses resultados, Min (2015) também aponta as dificuldades de mulheres que atuam no contexto operacional da construção em encontrar roupas e EPIs projetados especificamente para elas. De acordo com a autora, as trabalhadoras tendem a se deparar com a falta de alternativa e, assim, passam a utilizar EPIs e roupas ocupacionais que são feitos para homens.

Oo e Lim (2020) realizaram um levantamento estatístico através de ferramentas online no âmbito da Construção Civil da Austrália para identificar dificuldades relacionadas ao acesso de EPIs adequados por trabalhadoras. Nesse tema, os autores enfatizam que na Industria da Construção predomina a figura masculina, o que influencia nos entraves para que mulheres tenham acesso aos EPIs corretos. Desta forma, ficam evidenciados que os EPIs existentes para a Construção Civil australiana tendem a não se direcionar para as características físicas femininas.

Trazendo para destaque do estudo de Oo e Lim (2020) as implicações negativas referentes ao acesso e uso de EPIs por operárias australianas, tem-se os ajustes e alterações que as usuárias eram obrigadas a realizar nos produtos de proteção a elas oferecidos. Elevando os inconvenientes, também são citados a deficiência em treinamentos para o uso dos EPIs, o baixo conhecimento da questão da não conformidade de EPIs para mulheres por parte dos empregadores e as dificuldades que estes produtos ocasionavam durante a realização das atividades pelas trabalhadoras. Segundo os resultados dos autores, as calças e macacões enquanto vestimentas ocupacionais foram os mais citados como necessários de ajustes na opinião das trabalhadoras, principalmente no que se refere à redução de fundos e apertos em cinturas para serem adequadamente vestíveis.

\subsection{Síntese}

A partir das descrições das publicações, são percebidos pontos em comum que se direcionam para o aspecto da disparidade entre EPIs disponíveis e EPIs adequados para trabalhadoras da Construção Civil. Com isso, torna-se questionável se realmente existem produtos de proteção específicos para esse público e nesse ambiente de trabalho. Caso constatado que realmente não existam, pode surgir o questionamento de como esses EPIs devem ser para se caracterizarem como realmente femininos. Ademais, deve-se deixar enfatizado que as pesquisas abordadas também convergem para o aspecto do "ajuste" em EPIs "masculinos" para serem utilizados por mulheres. Posto isso, lança-se luz sobre esse tema para discussão no campo do Design.

Complementando o discorrido supra, Oo e Lim (2020) reforçam que o projeto e a fabricação de EPIs devem receber a devida atenção em virtude da sua importância em auxiliar na proteção dos trabalhadores a riscos, devendo isto ocorrer sem priorizar um ou outro gênero dos usuários. Conforme os autores, EPIs inadequadamente utilizados podem favorecer quedas de produtividade no ambiente laboral, lesões graves e, até mesmo, óbitos. Portanto, é necessária a revisão destes produtos. 


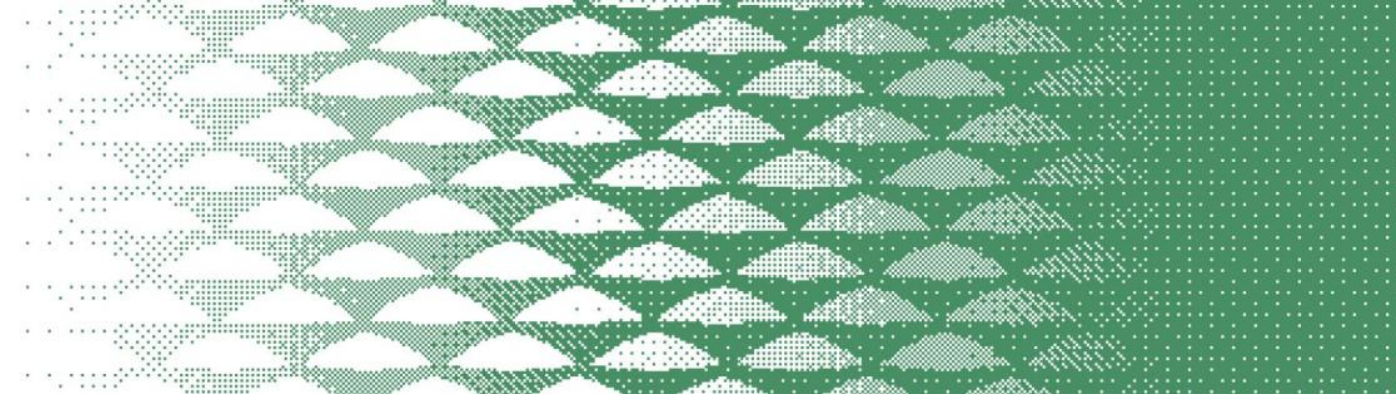

No que concerne à dificuldade de acesso de EPIs adequados para trabalhadoras da Construção Civil, Wagner, Kim e Gordon (2013) suscitam a recomendação de que os empregadores estimulem a compra e a disponibilização de produtos de proteção ocupacional realmente específicos para mulheres. Desta maneira, como apontam os autores, o mercado seria movimentado para a produção e comercialização desses produtos, facilitando o acesso dos EPIs corretos para ambos os gêneros.

Aproveitando a referência à comercialização, resgata-se a pesquisa de Min (2015), a qual destaca o duplo desafio das mulheres em relação aos EPIs em atividades operacionais: encontrar os EPIs conformes e se identificar no trabalho com o uso desses produtos. Por isso, é fundamental o cuidado na propagação de imagens publicitárias para não influenciar negativamente na autoimagem das mulheres enquanto trabalhadoras da Construção e setores relacionados, pois ainda resistem preconceitos sobre mulheres que atuam em meios onde os homens se preponderam em quantidade. Como se depreende do abordado pela autora, as mulheres devem se sentir realmente femininas, capazes e eficientes, o que não se percebe em perspectiva na realidade devido à pouca atenção fornecida à temática.

Corroborando com esse quadro de baixa atenção sobre o tema, Onyebeke et al. (2016) apontam que poucas são as pesquisas que enfocam as mulheres e a materialização de solução de suas necessidades em EPIs, o que também foi constatado por meio deste estudo. Para mudar a realidade atual das mulheres trabalhadoras da Construção Civil, que aparentemente estão pouco protegidas em termos de EPIs adequados e disponíveis para uso, entende-se que a problemática ainda deve ser ampliada para melhor análise e definição de ações.

De toda forma, através dos estudos abordados, fica nítido que discutir sobre EPIs para mulheres que trabalham na Construção Civil enseja que estas se envolvam direta ou indiretamente, principalmente em finalidades de subsidiar ações mais concretas na realidade. Isso tem embasamento no abordado por Rocha (2015), ao realçar a importância da escuta e da promoção do empoderamento das mulheres trabalhadoras para guiar ações estratégicas que lhes favoreçam melhores condições de trabalho.

\section{Considerações finais}

Através deste estudo baseado em revisão sistemática de literatura, foram identificados vários pontos de atenção quanto ao uso de EPIs por trabalhadoras da Construção, tornando alcançado o objetivo previamente formulado para a pesquisa. Destes pontos, ficou prevalecido o aspecto da inadequação antropométrica desses produtos devido ao desenvolvimento de EPIs historicamente voltado para o usuário homem. Adicionalmente, as publicações tendem a ressaltar que as mulheres precisam realizar ajustes nos produtos de proteção ocupacional para que possam usá-los em suas atividades de trabalho. Esses ajustes podem configurar constrangimentos e não garantem a segurança das usuárias.

Deve-se ressaltar o fato da relativa baixa quantidade de estudos incluídos nesta RSL, que foi em cinco para o intervalo de uma década. Com esse quadro, alerta-se para a necessidade de ampliação de estudos do tema dos EPIs para mulheres da construção civil, ocorrendo isso não apenas no Design, mas em todas as áreas do conhecimento em que o tema se insere. Em complemento, destaca-se que apenas um estudo brasileiro foi incluído para análise, mas os demais de outros países evidenciam que a questão do tema em abordagem pode ser global.

Posto isto, reforça-se a necessidade de ampliações e discussões sobre o tema das trabalhadoras da Construção Civil e os EPIs a elas disponíveis, pois, pelo que se percebe, consiste em um assunto consideravelmente silenciado por muitos anos. Fica demonstrada a demanda social em torno do tema e, por isso, defende-se a emergente ampliação da discussão tendo o Design como área de fundamento, principalmente devido à interdicisplinaridade que pode ser promovida, ao envolver Antropometria, Ergonomia, Design de Produto, Segurança do Trabalho, áreas sociais, dentre outros campos do saber. 
A partir do estudo aqui desenvolvido, deixa-se recomendado como estudos futuros novos levantamentos bibliográficos devido às limitações deste estudo a apenas três bases de dados de publicações científicas, termos de buscas, idiomas, dentre outros fatores delimitadores. Isto é necessário porque o abordado neste artigo não esgota o cenário da realidade em relação ao tema e nem o que há de estado da arte. Também são oportunas pesquisas que insiram as mulheres trabalhadoras da Construção Civil em uma abordagem participativa, colaborando diretamente com suas experiências, opiniões e, até mesmo, com sugestões tangíveis para os EPIs enquanto produtos.

Retomando a pergunta de pesquisa definida neste estudo, bem como os resultados alcançados, nota-se a necessidade da realização de mais estudos considerando o contexto brasileiro da atualidade quanto aos EPIs utilizados por trabalhadoras na Construção Civil. É importante verificar que até os aspectos normativos devem se tornar mais claros quanto ao atendimento da proteção das mulheres que reformam e constroem. Paralelamente, projetistas, fabricantes e fornecedores de EPIs devem participar da mudança de perspectiva para o desenvolvimento de EPIs como produtos efetivamente femininos. Portanto, nota-se que há várias lacunas a serem exploradas sobre EPIs para mulheres trabalhadoras da Construção Civil, que podem ser objeto de análise por meio de debates no campo do Design.

\section{PPE for working women in construction: an debate opportunity in Design}

Abstract: Historically, work stations were developed by and for men, principally in operational place as construction, and the the women insertion in the job market was late. Thus, there were repercussions on the development of Personal Protective Equipment (PPE), which tend to be based mostly on male characteristics. For this reason, there are still several obstacles to the access and use of adequate PPE by women workers in the construction, which is not just a national issue. In short, this paper is based on a Systematic Literature Review whose guiding question is what can be debated about the main barriers related to PPE as protection products used by women in construction? Furthermore, the main purpose is to raise inconsistencies and challenges with regard to PPE intended for use by construction workers female. Therefore, based on a review in three databases, five publications surveyed in the last ten years highlight the anthropometric inadequacy in PPE used by women, in addition to the need for product adjustments. However, there are other challenges for construction workers female that can foster timely debates in Design field.

Keywords: Design. Personal Protection Equipment. Construction female workers.

\section{Referências bibliográficas}

DEL CASTILLO, A. P. Personal protective equipment: getting the right fit for women. HesaMag, v. 12, p. 34-37, 2015.

DUL, J.; WEERDMEESTER, B. Ergonomia prática. Tradução: Itiro Iida. 3. ed. - São Paulo: Blucher, 2012.

ESCOLA NACIONAL DE INSPEÇÃO DO TRABALHO. NR 6 - Equipamento de Proteção Individual EPI. 2018. Disponível em: https://www.gov.br/trabalho/pt-br/inspecao/seguranca-e-saude-notrabalho/normas-regulamentadoras/nr-06.pdf. Acesso: maio. 2021. 
IIDA, I.; GUIMARÃES, L. B. M. Ergonomia: projeto e produção. 3. ed. São Paulo: Edgard Blucher, 2016.

MEIRELLES, L. A.; VEIGA, M. M.; DUARTE, F. A contaminação por agrotóxicos e o uso de EPI: análise de aspectos legais e de projeto. Laboreal, v. 12, n. 2, p. 75-82, 2016.

MESSING, K.; CATIGNY, C. Trabalho e gênero. In: FALZON, P. (Org.). Ergonomia. Tradução: Giliane M. J. Ingratta et al. 2 ed. São Paulo: Blucher, 2018.

MIN, S. Gendered role communication in marketing blue-collar occupational gear and clothing in the United States. Fashion and Textiles, v. 2, n. 1, p. 1-12, 2015.

OBREGON, R. F. A. Perspectivas de pesquisa em design: estudos com base na Revisão Sistemática de Literatura. Erechim: Deviant, 2017.

ONYEBEKE, Lynn C. et al. Access to properly fitting personal protective equipment for female construction workers. American Journal of Industrial Medicine, v. 59, n. 11, p. 1032-1040, 2016.

OO, B. L.; LIM, T. H. B. Women's Accessibility to Properly Fitting Personal Protective Clothing and Equipment in the Australian Construction Industry. In: IOP Conference Series: Earth and Environmental Science. IOP Publishing, 2020.

PANERO, J.; ZELNIK, M. Dimensionamento humano para espaços interiores: um livro de consulta e referência para projetos. Tradução: Anita Regina Di Marco. 1. ed. 4. reimp. Barcelona: Gustavo Gilli, 2008.

ROCHA, M. F. V. et al. Perfil de saúde laboral e auditiva de trabalhadoras e as ações afirmativas em indústrias do Paraná. Dissertação (Mestrado) - Universidade Tuiuti do Paraná, Curitiba, 2015.

ROSA, M.; QUIRINO, R. Relações de gênero e ergonomia: abordagem do trabalho da mulher operária. Holos, v. 5, p. 345-359, 2017.

SANTOS, A. Seleção do método de pesquisa: guia para pós-graduando em design e áreas afins. Curitiba: Insight, 2018.

WAGNER, H.; KIM, A. J.; GORDON, L. Relationship between personal protective equipment, selfefficacy, and job satisfaction of women in the building trades. Journal of Construction Engineering and Management, v. 139, n. 10, p. 04013005, 2013.

WANDERLEY, M. et al. Bases Comuns do Design: uma discussão sobre o impacto e papel social do design. In: ARRUDA, A. J. V. Design \& Complexidade. São Paulo: Blucher, 2017. 